\title{
BMAC and Adipose-Derived MSCs Treatment for Knee Osteoarthritis: A Systematic Review
}

\author{
Pooja Pithadia1* MSc, Sharmila Tulpule ${ }^{2}$ MBBS, M.S, MCh Ortho, Masud ur Rahman ${ }^{3}$ MBBS, FRCS, Mrinalini Singh ${ }^{4}$ PhD $^{1}$ \\ ${ }^{1}$ Medical Biotechnology, Medica Pain Management Clinic, London,UK. \\ ${ }^{2}$ Medica Stem Cells, Dubai,UAE. \\ ${ }^{3}$ Medica Stem Cells, Dublin, Ireland. \\ ${ }^{4}$ Medica Institute Pvt Ltd, India.
}

*Corresponding Author: Pooja Pithadia, Medical Biotechnology, Medica Pain Management Clinic, London,UK.

Received Date: 18 June 2021 | Accepted Date: 14 July 2021 | Published Date: 17 July 2021

Citation: P Pithadia, S Tulpule, M Rahman, M Singh. (2021) BMAC and Adipose-Derived MSCs Treatment for Knee Osteoarthritis: A Systematic Review. International Journal of Clinical Case Reports and Reviews. 7(4); DOI: 10.31579/2690-4861/150

Copyright: (C) 2021 Pooja Pithadia, This is an open-access article distributed under the terms of the Creative Commons Attribution License, which permits unrestricted use, distribution, and reproduction in any medium, provided the original author and source are credited.

\begin{abstract}
Background: Knee osteoarthritis is the most common musculoskeletal progressive disorder that affects nearly 303 million people worldwide. This condition prevails in $10 \%$ males and $13 \%$ females among the elders above 60 . Although there is conventional non-surgical and surgical treatment available for knee osteoarthritis, there is a fascinating interest in bone marrow as pirate concentrate (BMAC) as well as adipose-derived mesenchymal stem cells (AD-MSC), including enzymatically treated stromal vascular fraction (SVF) and mechanically treated (microfat/nanofat) injections among physicians. Hence, this systematic review aims to determine the efficacy of BMAC and AD-MSCs (enzyme and mechanically treated) injections for knee osteoarthritis treatment.

Methods: A systematic review was performed on the following data sources (PubMed, Scopus, Google Scholar, EMBASE, and Cochrane Library) published on March 31, 2021. The keywords or MeSH terms include 'Knee Osteoarthritis with 'Bone marrow aspirate concentrate' OR 'BMAC' or with 'Adipose-derived mesenchymal stem cells (AD-MSC)' or with 'Stromal vascular fraction' OR 'SVF' or 'Mechanically treated AD-MSC (mfat/nanofat)'. In addition, the retrieved articles were further reviewed to identify relevant research studies.

Results: The authors reviewed and tabulated data based on the year of study, study type, therapy protocol, patient population, outcome measures, and interpretation. Among the 382 records screened, 43 studies (16 on BMAC and 27 on AD-MSCs) were included in the systematic review study. Among them, only 5 were randomized controlled trials. These selected studies demonstrated short-term positive outcomes such as improvement in knee pain and function with no adverse side effects. Moreover, researchers reported varied administration methods of BMAC or AD-MSC either as standalone or in combination with other conservative procedures such as PRP (Platelets Rich Plasma), HA (Hyaluronic acid), or surgery.

Conclusions: BMAC and AD-MSC (enzymatically and mechanically treated) injections prove safer and more efficacious in patients with knee osteoarthritis for a shorter duration of 2 years. However, the available literature lacks high-quality studies with no varied clinical settings and long-term follow-up of more than two years.

Keywords: bmac; stromal vascular fraction; adipose-derived mesenchymal stem cells; bone marrow aspirate concentrate; svf; knee osteoarthritis
\end{abstract}

\section{Introduction}

Osteoarthritis (OA) is the most common type of progressive musculoskeletal arthritic disorder affecting nearly 303 million people worldwide [1]. Compared to all the joint regions, OA commonly affects hip and knee joints [2]. Due to a steady increase in ageing, obesity, and life expectancy, knee OA is prevalent in $10 \%$ males and $13 \%$ females among the elderly population [3].
Knee osteoarthritis (KOA) arises from gradual deterioration of the articular cartilage, changes to the subchondral bone, osteophyte formation, degeneration of menisci and ligaments, and inflammation of the adjacent tissues [4].

Patients were suffering from KOA experience chronic pain, swelling, stiffness, and limited range of motion in the affected joint, leading to a reduced quality of life [5]. 
The well-accepted first-line conservative options include RICE (Rest, Ice therapy, Compression, and Elevation) exercise, activity modification, and physiotherapy. As symptoms worsen, NSAIDs (non-steroidal antiinflammatory drugs), corticosteroids, and hyaluronic acid injections can relieve pain and improve joint function [6]. However, none of these treatments reverses or repair the degenerative nature of the disease [7]. Even the rapid disease progression to late-stage OA in patients who do not respond to conservative treatment would eventually require knee joint replacement [8].

In this scenario, there has been significant interest in developing efficacious conservative approaches classified as regenerative. Regenerative cell therapy uses the anti-inflammatory and healing properties of a patient's cells to treat inflamed and painful tissues [7] The use of Platelet Rich Plasma (PRP) and Prolotherapy are being evaluated to relieve the pain of OA $[9,10]$.

Recently, mesenchymal stem cells (MSCs) have appeared as a potential therapeutic regenerative option due to their ability of self-renewal, multilineage differentiation potential, immune-suppressive, antiapoptotic, anti-fibrotic, angiogenic, mitogenic, anti-inflammatory, and wound healing properties $[11,12]$. These MSCs are present in many adult tissues such as bone marrow, adipose tissues, articular cartilage, synovial membrane, periosteum, and the dermis [13] Among these sources, bone marrow mesenchymal stem cells (BMSCs) and adipose-derived mesenchymal stem cells (AD-MSCs) received more attention [14] ADMSCs are used in several forms, including stromal vascular fraction (SVF), culture-expanded adipose-derived stem cells, and minimally manipulated fat graft.

BMAC is obtained from the iliac crest via bone marrow needle aspiration, subsequently concentrated through dedicated centrifuges, and injected directly on the knee region [15]. Adipose tissue obtained through liposuction can be treated mechanically and enzymatically to extract adipose-derived mesenchymal stem cells (AD-MSCs). For mechanical extraction, adipose tissue was harvested mechanically in a closed system to extract the tissue-healing effect of micro-fragmented tissue [31]. For enzymatic extraction, collagenase is added to the non-enriched lipoaspirate, followed by its removal via a dilution step. In the dilution step, the lipid enzyme mixture is washed with normal saline followed by centrifugation. This final step extracts the SVF product, which can be directly administered to the patient [16].

This review aims to investigate the effectiveness of BMAC and ADMSCs (enzymatic and mechanically derived) injections regarding pain reduction and functional improvement in adult patients with knee osteoarthritis.

\section{Methods}

This systematic review was performed according to the PRISMA (Preferred Reporting Items for Systematic Reviews and Meta-Analyses) guidelines $[17,18]$.

A comprehensive, systematic literature search was performed in April 2021, and an analysis of these articles was conducted by all the authors involved in the study. The databases of PubMed, Scopus, Google Scholar, EMBASE, and Cochrane Library were searched from 2011 to March 31, 2021. The following keywords were used in different combinations: 'Knee Osteoarthritis with 'Bone marrow aspirate concentrate' OR 'BMAC' or 'Adipose-derived mesenchymal stem cells or 'Stromal vascular fraction' OR 'SVF' or 'Mechanically treated AD-MSC (mfat/nanofat)'.

\section{Study selection}

All participants in the trials had to have a clinical diagnosis of knee osteoarthritis under either intra-articular BMAC or AD-MSCs treatment.

We limited the search to articles in English, and only human studies were included. After assessing all titles and abstracts, all relevant articles were obtained. Even the bibliographies were also searched to identify further relevant literature that met our inclusion criteria.

All studies were included if their design could be classified into one of the following categories: open-label, randomized controlled trial, prospective, retrospective study, and pilot study.

We included studies in which adult participants were diagnosed with knee osteoarthritis by clinical or image evaluation. We excluded articles lacking access to the full text, conference presentations, narrative reviews, editorials, and expert opinions.

The articles found were pooled and subjected to inclusion and exclusion criteria established before the commencement of this systematic review. A PRISMA flowchart of this systematic review is provided in Figure I.

\section{Data extraction}

The researchers independently recorded the study design, therapy protocol, patient population, outcome measures, and interpretations. 


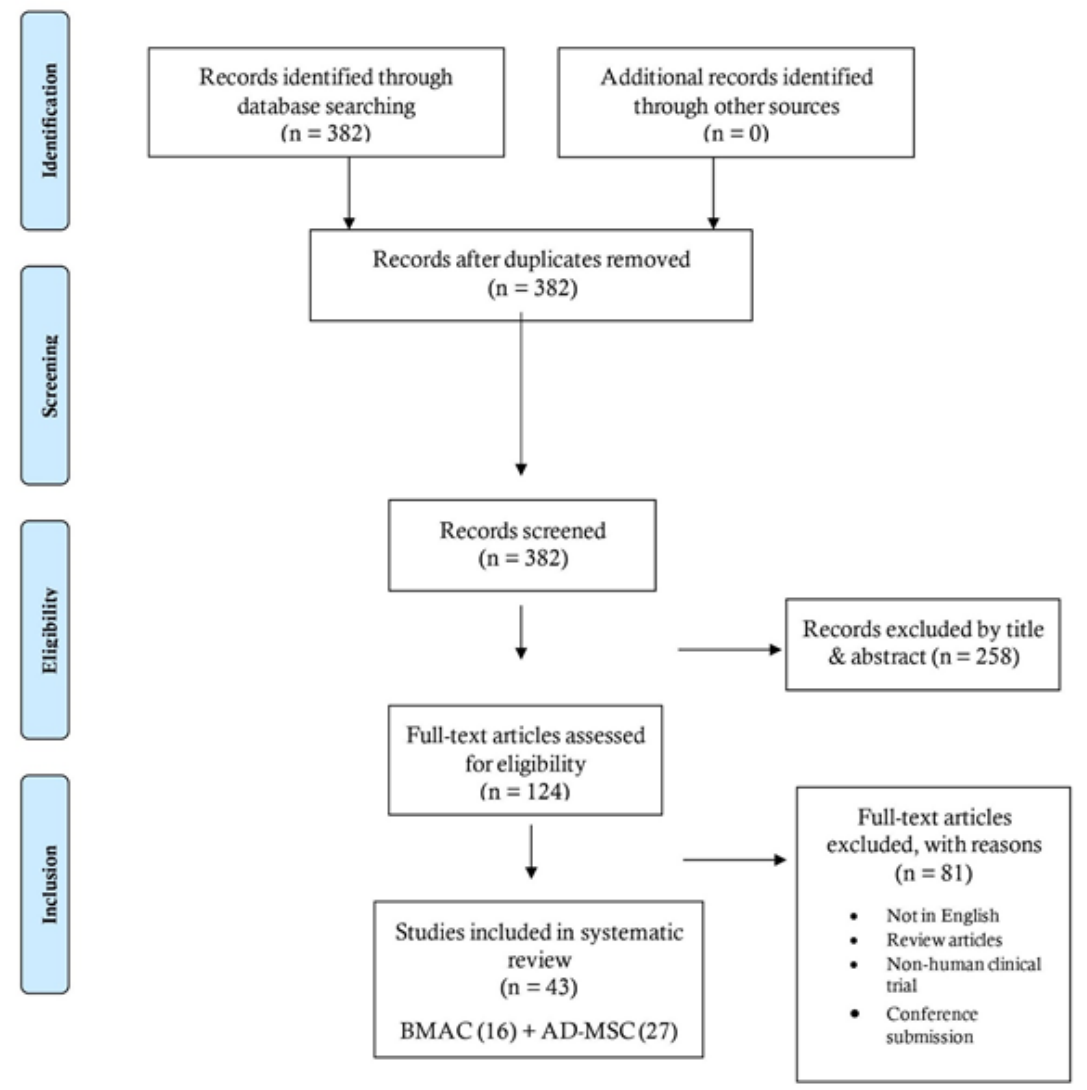

\section{Figure 1: A flow diagram of study inclusions: BMAC and AD-MSCs [PRISMA 2009 flow diagram]}

\section{Results}

\section{Literature search}

Of the 382 articles initially identified by the search, 16 [19,30,32,35] on BMAC and $27[36,62,16]$. on AD-MSCs, including SVF, met the inclusion criteria. Therefore, the relevant data is given in Tables III and IV.

\section{Participants}

The 16 studies under BMAC involved 10 to 681 patients with the age group of between 18-85 affected by knee OA [Table I), while 27 studies under AD-MSCs, including SVF, involved 2 to 2586 knee OA patients between 18-89 age group [Table II]. Among these 44 studies, only 5 were randomized controlled trials. Fourteen papers were prospective studies, with three of them being comparative, two being open-label, one being a pilot study. The rest were retrospective studies, with two of them being comparative.

\section{Therapeutic approaches}

Regarding the therapeutic protocol, BMAC was either injected alone or combined with PRP in the same session, alternatively as a booster dose after a certain period. Very few authors injected BMAC in association with adipose tissue or scaffold. Under AD-MSCs, it was either injected alone or combined with PRP, adipose tissue, HA, or scaffold.

\section{Outcome measures}

Regarding outcomes, varied clinical scores such as WOMAC, VAS, KOOS, IKDC, KSS, ICOAP, NPS, and LEFS were used to evaluate the outcomes of BMAC injections (Table 1) and AD-MSCs injections (Table 2). Even MRI was performed before and after the procedure to detect positive changes in the resultant images. Very few authors used ICRS, OKS, NRS, ROM, Tegner activity, Lysholm patient satisfaction scores, and PROMIS questionnaires. Immunohistochemical analysis was reported only in Roato et al. 55. 's study involving AD-MSCs injections.

Table I: Patients' demographics [BMAC]

\begin{tabular}{|c|c|c|c|c|}
\hline Articles & Total enrolled & M/F & Age group & KL grade \\
\hline 19Shapiro et al., 2017 & 25 & $7 / 18$ & - & - \\
\hline 20Shapiro et al., 2018 & 25 & $7 / 18$ & $42-68$ & I-II \\
\hline 21Kim et al., 2014 & 41 & $17 / 24$ & $53-80$ & I-IV \\
\hline 22Sampson et al., 2016 & 73 & - & $23-79$ & III-IV \\
\hline 23Krych et al, 2016 & 46 & $23: 15 / 8$ & Mean 38 & - \\
& $(23+12+11)$ & $12: 8 / 4$ & & \\
& & $11: 8 / 3$ & & $18-80$ \\
\hline 24Anz et al., 2020 & 90 & - & I-III \\
\hline
\end{tabular}




\begin{tabular}{|c|c|c|c|c|}
\hline 25Centeno et al, 2014 & $\begin{array}{c}681 \\
(616 \text { vs } 224)\end{array}$ & $\begin{array}{c}616: 397 / 219 \\
224: 119 / 105\end{array}$ & 54.3 vs. 59.9 & I-IV \\
\hline 26Centeno et al, 2015 & $\begin{array}{c}373 \\
(224 \text { vs } 185)\end{array}$ & $\begin{array}{c}224: 143 / 81 \\
185: 140 / 45\end{array}$ & 54.5 vs. 50.2 & I-IV \\
\hline 27Rodriguez et al, 2018 & 19 & $3 / 16$ & $58(30-80)$ & I-II \\
\hline $\begin{array}{c}\text { 28Themistocleous et al., } \\
\text { 2018 }\end{array}$ & 121 & $36 / 85$ & $70(50-85)$ & III-IV \\
\hline 29Ryu et al., 2020 & 52 & - & - & - \\
\hline 30Kristin et al., 2015 & 70 & - & - & II-IV \\
\hline 32Oliver et al., 2015 & 70 & $21 / 49$ & - & II-IV \\
\hline 33Shaw et al., 2018 & 15 & $5 / 10$ & Mean 67.7 & - \\
\hline 34Vad et al, 2016 & 10 & $4 / 6$ & $63.5(52-73)$ & III-IV \\
\hline 35Hernigou et al, 2018 & 30 & $12 / 18$ & $28(18-41)$ & IV \\
\hline
\end{tabular}

Table II: Patients' demographics [AD-MSCs]

\begin{tabular}{|c|c|c|c|c|}
\hline Articles & Total enrolled & $\mathbf{M} / \mathbf{F}$ & Age group & KL grade \\
\hline 36Gibbs et al., 2015 & 4 & $2 / 2$ & $23-50$ & - \\
\hline 37Bansal et al., 2017 & 10 & - & $\geq 50$ & I-II \\
\hline 7Fodor et al., 2016 & 6 & - & $51-69$ & I-III \\
\hline 38Garza et al., 2015 & 6 & - & $59(52-69)$ & II-III \\
\hline 39Hong et al., 2019 & 16 & - & $18-70$ & II-III \\
\hline 40Mautner et al., 2019 & 110 & $\begin{array}{l}24 / 17 \\
12 / 23\end{array}$ & $\begin{array}{l}59 \pm 11 \\
63 \pm 11\end{array}$ & - \\
\hline 41Pak J, 2011 & 2 & - & $60-87$ & - \\
\hline 42Pak et al., 2013 & 74 & - & - & - \\
\hline 43Pak et al., 2016 & 3 & - & $60-87$ & III \\
\hline 44Pintat et al., 2017 & 19 & $10 / 9$ & - & - \\
\hline 45Yokota et al., 2017 & 13 & $2 / 11$ & 74.5 & III-IV \\
\hline 46Hudetz et al., 2017 & 17 & $12 / 5$ & $40-85$ & III-IV \\
\hline 47Pers et al., 2016 & 18 & - & $50-75$ & III-IV \\
\hline 48Berman et al, 2019 & 2,586 & - & - & - \\
\hline 16Zhang et al., 2021 & 47 (29 vs 24$)$ & - & - & II-III \\
\hline $\begin{array}{c}\text { 49Lapuente et al., } \\
2020\end{array}$ & 50 & - & $50-89$ & - \\
\hline 50Simunec et al, 2020 & 12 & $5 / 7$ & $61(51-80)$ & III-IV \\
\hline 51Koh et al, 2013 & 18 & $6 / 12$ & $54.6(41-69)$ & - \\
\hline 52Koh et al., 2014 & 44 (23 vs 21$)$ & - & - & - \\
\hline 53Koh et al., 2014 & 37 knees & - & $57.4(48-69)$ & - \\
\hline 54Koh et al., 2015 & 30 & - & - & - \\
\hline 55Roato et al, 2019 & 20 & $9 / 11$ & 59.6 & I-III \\
\hline 56Jones et al., 2018 & 54 (27 vs 27$)$ & - & - & - \\
\hline 57Bui et al., 2014 & 21 & - & $\leq 18$ & II-II \\
\hline 58Nguyen et al, 2017 & 30 (15 vs. 15$)$ & $3 / 12$ vs $3 / 12$ & 58.60 vs. 58.20 & II-III \\
\hline 59Kim et al., 2015 & 49 (55 knees) & - & - & I-II \\
\hline 60Kim et al., 2015 & $\begin{array}{c}54 \text { (56 knees): } \\
37 \text { (39 knees) vs } 17 \\
\text { (17 knees) }\end{array}$ & - & - & - \\
\hline
\end{tabular}

Table III: Clinical studies regarding the use of BMAC to treat knee osteoarthritis

\begin{tabular}{|c|c|c|c|c|c|}
\hline Ref & Study & Therapy protocol & Outcome & $\begin{array}{c}\text { Follow up } \\
\text { (mon) }\end{array}$ & Conclusion \\
\hline [36] & Case series & $\begin{array}{c}\text { SVF + PRP + } \\
\text { moderate exercise } \\
\text { for 4 months }\end{array}$ & $\begin{array}{c}\text { KOOS Physical function } \\
\text { tests: GUG, SCT RPE }\end{array}$ & 12 & Less Pain \& better knee function \\
\hline$[37]$ & Prospective & SVF + PRP & $\begin{array}{c}\text { WOMAC, 6-minute walking } \\
\text { distance, MRI }\end{array}$ & $\begin{array}{c}\text { Significant improvement of WOMAC scores } \\
\text { and 6-minute walking distance. MRI showed } \\
\text { increase in cartilage thickness in all but 2 } \\
\text { patients. All patients are satisfied with therapy. }\end{array}$ \\
\hline
\end{tabular}




\begin{tabular}{|c|c|c|c|c|c|}
\hline Ref & Study & Therapy protocol & Outcome measures & Follow up (mon) & Conclusion \\
\hline [19] & $\begin{array}{l}\text { Single-blind, } \\
\text { prospective } \\
\text { RCT }\end{array}$ & $\begin{array}{l}\text { BMAC + Platelet- } \\
\text { poor bone marrow } \\
\text { plasma vs. saline }\end{array}$ & $\begin{array}{l}\text { VAS, ICOAP, WOMAC, } \\
\text { KOOS }\end{array}$ & 6 & No significant improvement \\
\hline$[20]$ & $\begin{array}{l}\text { Single-blind } \\
\text { RCT }\end{array}$ & $\begin{array}{c}\text { BMAC + } \\
\text { Platelet poor } \\
\text { plasma vs. saline } \\
\end{array}$ & VAS, ICOAP, algometer & 12 & $\begin{array}{l}\text { Significant improvement in pain \& QoL. } \\
\text { No superiority to saline. } \\
\text { MRI - No cartilage regeneration }\end{array}$ \\
\hline$[21]$ & Retrospective & $\begin{array}{c}\text { BMAC+ } \\
\text { adipose tissue inj. }\end{array}$ & $\begin{array}{l}\text { VAS, IKDC, SF-36, KOOS, } \\
\text { Lysholm }\end{array}$ & 8.7 & $\begin{array}{l}\text { Significant improvement of pain \& } \\
\text { function. }\end{array}$ \\
\hline$[22]$ & Retrospective & $\begin{array}{l}\text { BMAC followed by } \\
\text { PRP at 8th week }\end{array}$ & $\begin{array}{l}\text { VAS, global patient } \\
\text { satisfaction score }\end{array}$ & 5 & $\begin{array}{l}\text { Significant improvement of pain with high } \\
\text { patient satisfaction }\end{array}$ \\
\hline$[23]$ & $\begin{array}{l}\text { Cohort, } \\
\text { prospective }\end{array}$ & $\begin{array}{c}\text { Scaffold + PRP } \\
\text { vs } \\
\text { scaffold + BMAC } \\
\text { vs } \\
\text { control scaffold }\end{array}$ & MRI & 12 & $\begin{array}{l}\text { Improved cartilage maturation with greater } \\
\text { fill and mean } \mathrm{T} 2 \text { values closer to that of } \\
\text { superficial native hyaline cartilage }\end{array}$ \\
\hline$[24]$ & RCT & $\begin{array}{c}\text { BMAC vs } \\
\text { leukocyte rich PRP }\end{array}$ & WOMAC, IKDC & $\begin{array}{l}1,3,6,9, \& 12 \\
\text { before \& after }\end{array}$ & $\begin{array}{c}\text { PRP \& BMC were effective in improving } \\
\text { patient-reported outcomes; neither } \\
\text { treatment provided a superior benefit }\end{array}$ \\
\hline$[25]$ & $\begin{array}{l}\text { Comparative } \\
\text { retrospective } \\
\text { Group A vs B }\end{array}$ & $\begin{array}{l}\text { (A) BMAC+PRP } \\
\text { vs. (B) } \\
\text { BMAC+PRP+ } \\
\text { adipose graft }\end{array}$ & $\begin{array}{l}\text { NPS, LEFS, improvement } \\
\text { rating score }\end{array}$ & $6-10$ & $\begin{array}{l}\text { Significant improvement of pain and } \\
\text { function. } \\
\text { No significant benefit with the addition of } \\
\text { adipose graft to BMAC. }\end{array}$ \\
\hline$[26]$ & $\begin{array}{l}\text { Comparative } \\
\text { retrospective } \\
\text { Group A vs B }\end{array}$ & $\begin{array}{c}\text { A- } 4 \times 108 \text { cells } \\
\text { BMAC+PRP } \\
\text { vs } \\
\text { B- }>4 \times 108 \text { cells } \\
\text { BMAC+PRP } \\
\end{array}$ & $\begin{array}{l}\text { NPS, LEFS, IKDC, } \\
\text { improvement rating score }\end{array}$ & $3-15$ & $\begin{array}{l}\text { Significant improvement of pain and } \\
\text { function. Significantly higher pain } \\
\text { reduction with high cell content. }\end{array}$ \\
\hline$[27]$ & Retrospective & BMAC only & $\begin{array}{l}\text { WOMAC \& Satisfaction } \\
\text { rate score }\end{array}$ & $6-24$ & $\begin{array}{l}\text { Better WOMAC score. } \\
\text { No significant difference between 6-month } \\
\text { and latest follow-up scores. } \\
\text { Variable satisfaction rate (63.2\% yes, } \\
36.8 \% \text { no). }\end{array}$ \\
\hline$[28]$ & Retrospective & BMAC only & NPS \& OKS & 11 & $\begin{array}{l}\text { Significant improvement of pain \& } \\
\text { function }\end{array}$ \\
\hline [29] & Retrospective & $\begin{array}{c}\text { BMAC } \\
\text { vs } \\
\text { hUCB-MSCs }\end{array}$ & $\begin{array}{l}\text { VAS, IKDC, KOOS, M- } \\
\text { MOCART, \& ICRS }\end{array}$ & 24 & $\begin{array}{l}\text { Significantly improvement in all outcomes } \\
\text { in both groups; but no differences between } \\
\text { two groups }\end{array}$ \\
\hline$[30]$ & $\begin{array}{l}\text { Prospective } \\
\text { case series }\end{array}$ & BMAC only & Adverse events, KOOS & Baseline, $3, \& 6$ & $\begin{array}{l}\text { Transient pain and swelling. Positive } \\
\text { KOOS with improved pain, QoL, daily } \\
\text { activities, \& sports/recreation score } \\
\text { without major complication }\end{array}$ \\
\hline$[32]$ & $\begin{array}{l}\text { Prospective } \\
\text { case series }\end{array}$ & $\mathrm{BMAC}+\mathrm{SVF}$ & Adverse events, KOOS & Baseline, 3, \& 6 & $\begin{array}{l}\text { Transient pain and swelling. Positive } \\
\text { KOOS with improved pain, QoL, daily } \\
\text { activities, \& sports/recreation score } \\
\text { without major complication }\end{array}$ \\
\hline [33] & Retrospective & $\begin{array}{l}4 \text { sequential BMAC } \\
\text { injections in } 3 \\
\text { months }\end{array}$ & $\begin{array}{l}\text { Resting/active NPS, overall } \\
\text { percentage improvement \& } \\
\text { LEFS }\end{array}$ & 24 days & $\begin{array}{l}\text { Significant improvement of pain \& } \\
\text { function. } \\
\text { Multiple injections are more effective than } \\
\text { a single one. }\end{array}$ \\
\hline [34] & Pilot trial & BMAC only & MRI, WOMAC, NRS & $\begin{array}{c}14 \\
(13-15)\end{array}$ & $\begin{array}{l}\text { Significant improvement in WOMAC and } \\
\text { NRS scores. } \\
\text { MRI - increase in extracellular matrix } \\
\text { thickness by an average of } 14 \% \text {. } \\
\text { Better improvement for patients younger } \\
\text { than } 63.5 \text { years old. }\end{array}$ \\
\hline$[35]$ & $\mathrm{RCT}$ & BMAC vs TKA & $\begin{array}{l}\text { MRI, bone marrow lesion } \\
\text { volume, Knee society score }\end{array}$ & $12(8-16)$ years & $\begin{array}{c}\text { Decrease in lesion size by } 40 \% \text { with better } \\
\text { cartilage and bone repair. } \\
\text { No significant difference in outcomes } \\
\text { between BMAC \& TKA. Majority } \\
\text { preferred BMAC. }\end{array}$ \\
\hline
\end{tabular}




\begin{tabular}{|c|c|c|c|c|c|}
\hline [7] & $\begin{array}{l}\text { Phase I open } \\
\text { label single- } \\
\text { arm }\end{array}$ & SVF & $\begin{array}{l}\text { WOMAC, VAS, ROM, OA } \\
\text { index, knee motion, timed up- } \\
\text { and-go (TUG), \& MRI }\end{array}$ & 12 & $\begin{array}{l}\text { No infections, acute pain flares, or other } \\
\text { adverse events. } \\
\text { significant improvement in WOMAC, VAS, } \\
\text { ROM \& TUG. } \\
\text { MRI- no detectable structural differences. } \\
\text { Full activity with decreased knee pain }\end{array}$ \\
\hline$[38]$ & $\begin{array}{l}\text { Feasibility \& } \\
\text { safety study }\end{array}$ & SVF & $\begin{array}{l}\text { PROMIS questionnaire, pain } \\
\text { \& mobility questionnaire }\end{array}$ & $\begin{array}{l}2,4,6, \& 12 \\
\text { weeks }\end{array}$ & $\begin{array}{l}\text { Decreased pain and increased mobility with no } \\
\text { side effects }\end{array}$ \\
\hline [39] & $\begin{array}{l}\text { Double-blind } \\
\text { RCT }\end{array}$ & $\begin{array}{l}\text { SVF vs HA. } \\
\text { Bilateral OA }\end{array}$ & $\begin{array}{l}\text { VAS, WOMAC, ROM, } \\
\text { whole-organ MRI score }\end{array}$ & 12 & $\begin{array}{l}\text { VAS, WOMAC, \& ROM improved } \\
\text { significantly for both groups, but these } \\
\text { improvements were not long lasting in the } \\
\text { control group. } \\
\text { MRI - significantly increased cartilage repair in } \\
\text { the SVF group compared to the control. }\end{array}$ \\
\hline$[40]$ & Retrospective & MFAT vs BMAC & KOOS, EQOL, VAS & 6 & $\begin{array}{l}\text { Significant improvement in pain and function, } \\
\text { EQOL, VAS, \& KOOS with both treatments, } \\
\text { with no significant difference between them. }\end{array}$ \\
\hline [41] & Case series & $\begin{array}{l}\mathrm{SVF}+\mathrm{PRP}+\mathrm{HA}+ \\
\text { Calcium chloride }+ \\
\text { 1ng dexamethasone }\end{array}$ & $\begin{array}{l}\text { VAS, Knee motion range, } \\
\text { Functional rating index, MRI }\end{array}$ & 3 & Improvement in pain \& knee function \\
\hline$[42]$ & Safety study & SVF + PRP & VAS, MRI & 12 & $\begin{array}{c}\text { Safe with no adverse side effects. Improvement } \\
\text { in VAS \& cartilage repair }\end{array}$ \\
\hline [43] & Case series & $\begin{array}{l}\mathrm{SVF}+\mathrm{PRP}+\mathrm{HA}+ \\
\text { Calcium chloride }\end{array}$ & $\begin{array}{c}\text { VAS, Knee motion range, } \\
\text { functional rating index, MRI }\end{array}$ & 5 & $\begin{array}{l}\text { Safe with improvement in pain and knee } \\
\text { function }\end{array}$ \\
\hline [44] & Prospective & AD-MSC+ PRP & WOMAC, MRI, \& ICRS & 12 & $\begin{array}{l}\text { Improvement in WOMAC \& cartilage repair } \\
\text { with no adverse side effects }\end{array}$ \\
\hline [45] & Prospective & SVF & VAS, WOMAC, JKOM & 6 & $\begin{array}{l}\text { VAS, WOMAC, \& JKOM improved } \\
\text { significantly }\end{array}$ \\
\hline$[46]$ & Prospective & MFAT & $\begin{array}{l}\text { VAS, dGEMRIC MRI, IgG } \\
\text { isolation from plasma and } \\
\text { synovial fluid }\end{array}$ & 12 & $\begin{array}{c}\text { Significant decrease in VAS scores. No change } \\
\text { in IgG. } \\
\text { MRI displayed increase in proteoglycan } \\
\text { content within the ECM. }\end{array}$ \\
\hline [47] & $\begin{array}{l}\text { Phase I } \\
\text { multicentric, } \\
\text { prospective, } \\
\text { single-arm, } \\
\text { open-label, } \\
\quad \text { dose } \\
\text { escalating }\end{array}$ & $\begin{array}{l}\text { SVF injection with } 3 \\
\text { varied stromal cell } \\
\text { doses } \\
2 \times 10610 \times 106 \\
50 \times 106\end{array}$ & $\begin{array}{l}\text { VAS, WOMAC, OA index } \\
\text { Patient global assessment } \\
\text { Knee injury, OA outcome } \\
\text { score, short arthritis } \\
\text { assessment scale SF-36 } \\
\text { quality-of-life questionnaire }\end{array}$ & 6 & $\begin{array}{l}\text { Less pain and better knee function only in the } \\
\text { low-dose group }\end{array}$ \\
\hline$[48]$ & Prospective & SVF + PRP & $\begin{array}{l}\text { VAS, WOMAC, adverse } \\
\text { events score }\end{array}$ & $12 \& 24$ & $\begin{array}{c}\text { No difference in outcomes between SVF alone } \\
\text { or with PRP added to SVF. } \\
\text { Very few minor side effects. } \\
\text { Less pain and greater ease of mobility. } 82 \% \\
\text { overall improvement }\end{array}$ \\
\hline [16] & Clinical trial & SVF & $\begin{array}{l}\text { WOMAC, VAS, ROM, } \\
\text { WORMS, \& MOCART }\end{array}$ & $\begin{array}{l}\text { before \& after } \\
1-, 3-, 6-, \& 12\end{array}$ & $\begin{array}{c}\text { WOMAC, VAS, ROM - significant } \\
\text { improvement. } \\
\text { MRI - thickness, volume, surface of cartilage } \\
\text { defect decreased. } \\
\text { WORMS \& MOCART - improvement in } \\
\text { cartilage repair with no adverse side effects }\end{array}$ \\
\hline [49] & Retrospective & SVF & $\begin{array}{l}\text { Lequesne, WOMAC, VAS, } \\
\text { quantification of the } \\
\text { biochemical profiles of } \\
\text { synovial fluid }\end{array}$ & 12 & $\begin{array}{c}\text { Safe \& effective with no adverse effects. } \\
\text { Significant improvement in all scores after 1- } \\
\text { year follow-up for all ages \& OA degree } \\
\text { groups. }\end{array}$ \\
\hline$[50]$ & $\begin{array}{l}\text { Comparative } \\
\text { case series }\end{array}$ & $\begin{array}{l}\text { SVF+PRP vs SVF } \\
\text { only }\end{array}$ & KOOS \& MRI & 12 & $\begin{array}{l}\text { Significant improvement KOOS in } 3 \text { of the } 4 \\
\text { treatment groups. } \\
67 \% \text { of the patients were satisfied or very } \\
\text { satisfied with the procedure and would } \\
\text { recommend it to others. } \\
\text { No serious adverse events }\end{array}$ \\
\hline [51] & Case series & $\begin{array}{l}\text { infrapatellar fat pad } \\
\text { derived MSC + PRP }\end{array}$ & $\begin{array}{l}\text { Lysholm score, VAS, MRI, } \\
\text { OA Index, WOMAC }\end{array}$ & $24.3(24-26)$ & $\begin{array}{l}\text { Significant improvement in all these scores. } \\
\text { Effective for reducing pain \& improving knee } \\
\text { function }\end{array}$ \\
\hline
\end{tabular}




\begin{tabular}{|c|c|c|c|c|c|}
\hline$[52]$ & $\begin{array}{c}\text { Prospective, } \\
\text { comparative } \\
\text { observational } \\
\text { study }\end{array}$ & $\begin{array}{c}\mathrm{HTO}+\mathrm{PRP} \\
\text { Vs } \\
\mathrm{HTO}+\mathrm{PRP}+\mathrm{SVF}\end{array}$ & Lysholm score, KOOS, VAS & & $\begin{array}{c}\text { PRP + SVF showed improved cartilage } \\
\text { healing, better KOOS, \& VAS score when } \\
\text { compared with PRP only }\end{array}$ \\
\hline$[53]$ & $\begin{array}{l}\text { Retrospective } \\
\text { Case series }\end{array}$ & AD-MSC & $\begin{array}{l}\text { IKDC, Tegner activity scale, } \\
\text { cartilage repair using ICRS } \\
\text { grading }\end{array}$ & $26.5(24-34)$ & $\begin{array}{l}\text { Improvement in all scores with encouraging } \\
\text { outcomes in cartilage repair }\end{array}$ \\
\hline [54] & $\begin{array}{l}\text { Therapeutic } \\
\text { case series }\end{array}$ & SVF + arthro. lavage & KOOS, VAS, Lysholm score & $\begin{array}{l}\text { Before and } \\
\text { after } 3,12, \& \\
24\end{array}$ & $\begin{array}{c}\text { Almost all patients showed significant } \\
\text { improvement in all clinical outcomes at the } \\
\text { final follow-up examination. } \\
\text { None of the patients underwent TKS during } \\
\text { this } 2 \text {-year period. } \\
\text { Adipose-derived SVF - good option in elderly } \\
\text { patients }\end{array}$ \\
\hline [55] & Prospective & $\begin{array}{l}\text { autologous conc. } \\
\text { adipose tissue after } \\
\text { lipoaspirate } \\
\text { centrifugation }\end{array}$ & $\begin{array}{l}\text { WOMAC, VAS, MRI, } \\
\text { immunohistochemistry }\end{array}$ & 18 & $\begin{array}{c}\text { Both WOMAC \& VAS scores improved } \\
\text { significantly, WOMAC showed progressively } \\
\text { better outcomes. } \\
\text { MRI: Outerbridge grade did not show } \\
\text { significant changes. Immunohistochemistry } \\
\text { displayed new tissue growth. }\end{array}$ \\
\hline$[56]$ & $\begin{array}{l}\text { Comparative } \\
\text { prospective, } \\
\text { single-center, } \\
\text { parallel-group } \\
\text { RCT }\end{array}$ & SVF vs HA & $\begin{array}{c}\text { WOMAC, PROMIS } \\
\text { questionnaire, synovial fluid } \\
\text { analysis, sway velocity } \\
\text { assessment }\end{array}$ & 6 & Ongoing \\
\hline [57] & Prospective & $\mathrm{SVF}+\mathrm{PRP}$ & VAS, Lysholm scores, MRI & 6 & $\begin{array}{l}\text { Significant improvement in VAS \& Lysholm } \\
\text { scores. } \\
\text { MRI analysis showed partial regeneration \& } \\
\text { thickening of articular cartilage }\end{array}$ \\
\hline$[58]$ & $\begin{array}{l}\text { Comparative } \\
\text { prospective }\end{array}$ & $\begin{array}{c}\mathrm{AM}+ \\
\mathrm{SVF}+\mathrm{PRP} \text { injection } \\
\text { Vs. AM alone }\end{array}$ & $\begin{array}{l}\text { WOMAC, VAS, Lysholm } \\
\text { scores, MRI, knee joint } \\
\text { function }\end{array}$ & 18 & $\begin{array}{l}\text { WOMAC, Lysholm, \& VAS scores improved } \\
\text { for both groups up to } 12 \text { months, but at } 18 \\
\text { months, the SVF group was significantly better } \\
\text { than the control group. } \\
\text { At } 12 \text { months, the SVF group displayed } \\
\text { significantly less bone marrow edema than the } \\
\text { control group. }\end{array}$ \\
\hline [59] & $\begin{array}{c}\text { Case series } \\
\text { retrospective }\end{array}$ & AD-MSC & $\begin{array}{c}\text { IKDC, Tegner activity score, } \\
\text { patients' overall satisfaction } \\
\text { score }\end{array}$ & - & $\begin{array}{l}\text { Significant improvement in all scores. The } \\
\text { clinical outcomes of MSC implantation for } \\
\text { knee OA are encouraging. }\end{array}$ \\
\hline$[60]$ & $\begin{array}{l}\text { Cohort } \\
\text { study }\end{array}$ & $\begin{array}{l}\text { MSCs loaded as a } \\
\text { scaffold } \\
\text { vs MSC without } \\
\text { scaffold }\end{array}$ & $\begin{array}{l}\text { IKDC, Tegner activity scale, } \\
\text { cartilage repair assessed with } \\
\text { ICRS grade }\end{array}$ & $28.6(24-34)$ & $\begin{array}{c}\text { Clinical \& arthroscopic outcomes of MSC } \\
\text { implant were encouraging in both groups, } \\
\text { although there were no significant differences } \\
\text { between groups. However, second-look } \\
\text { arthroscopy showed better ICRS grades in } \\
\text { Group } 2 \text {. }\end{array}$ \\
\hline
\end{tabular}

Table IV: Clinical studies regarding the use of AD-MSCs to treat knee osteoarthritis

\section{Safety and efficacy of BMAC and AD-MSCs therapy}

None of the studies analyzed in this systematic review recorded any complication or adverse effect of BMAC and AD-MSCs administration. Only mild pain and swelling have been observed in very few patients within the initial few days following BMAC/AD-MSCs injection procedure. Furthermore, both BMAC and AD-MSCs showed positive clinical outcomes with significant improvement in pain, articular function, and range of movement.

\section{Discussion}

The results of this systematic review validate that both BMAC and ADMSCs treatments are safe and effective to treat knee OA. However, the therapeutic use of BMAC and AD-MSCs, especially SVF, is restricted across the United States, Europe, and many other countries based on safety and efficacy concerns.
The significant finding of this systematic review is that most of the studies are of low quality with a lack of well-defined methodologies, with very few RCTs, thus preventing us from providing any substantial conclusions on the therapeutic potential of these AD-MSCs and BMAC injections.

Furthermore, there is an inadequate patient selection process, although these studies reported good reliability. The inclusion and exclusion criteria, recruitment rate, and a well-defined selection process were rarely reported. Hence, further studies including larger patient cohorts should be performed to demonstrate the long-term effect of both BMAC and ADMSCs injections.

Many patients underwent conservative treatments such as steroid treatment or surgical procedures in most of these studies, such as microfracture, arthroscopic debridement, or high tibial osteotomy. Hence there is no clear understanding of the exclusive clinical potential of these BMAC and AD-MSCs injections. 
We can find the release of platelet-rich plasma (PRP) treatment without adequate evidence in the recent past. This treatment has been used clinically due to high media exposure only [61]. There is a possibility to exempt 510(k) regulations [62]. New medical devices "substantially equivalent" to those already prevalent in the market can skip the standard FDA approval process. Hence, there was an increase in the production of PRP kits. However, this market saturated due to overproduction by various preparation systems, thereby preventing a "standardization" of PRP therapy for knee OA treatment.

This same scenario is now approaching AD-MSCs and BMAC therapies that are not affected by the regulatory burden. Moreover, they can be quickly harvested from the OA patient and administered immediately through an intra-articular injection with PRP or HA (hyaluronic acid). HA provides an environment where MSCs can easily adhere to the target area around the lesion and differentiate into cells to build damaged bone and cartilage. Similarly, PRP consists of highly concentrated platelets and varied growth factors to exacerbate the proliferation of MSCs $[68,69]$. Hence, this simultaneous use of other biological agents or administering these treatments following the conventional procedures prevent a reasonable comparison of the studies performed so far.

The available RCTs have several biases since most of the patients were treated bilaterally $[20,63]$. This is not the ideal condition to determine the efficacy of a treatment since the patients cannot evaluate one knee independently from the other. There was no proper clarity on the number of cells administered and the exact number of injections for the best outcome. It was even difficult to interpret which one of the two treatments provide better outcomes. Although their immunophenotypes are more than $90 \%$ identical $[64,65]$, they still have many distinct characteristics, especially in their cell surface markers, differentiation potentials, and distribution within the body. An in vitro analysis revealed that almost 300fold more SVF can be derived from $100 \mathrm{~g}$ of adipose tissue when compared to $100 \mathrm{ml}$ of bone marrow aspirate $[66,67]$. However, there is no apparent connection between the quantity and the dose-effect. Furthermore, there is no substantial evidence to define the patient's profile that could respond better to a specific treatment compared to others. Hence, this topic demands more research to understand the effect of both BMAC and AD-MSCs therapies.

Both bone marrow harvesting and lipoaspiration are minimally invasive procedures with minimal side effects. However, lipoaspiration was more severe due to the associated risks of pain and hematoma. Anyway, the surgeon who opts for these treatments depends on the availability of preparation kits in different countries. Moreover, industries have been releasing their proprietary kits for BMAC and AD-MSCs preparation, with new methods still being developed. However, there is no adequate research evidence to support the ability of MSCs.

At present, stem cell treatment is expensive and cannot be considered a "routine" treatment for knee cartilage degeneration. From a clinical viewpoint, the use of BMAC and AD-MSCs for knee OA treatment seems to be safe and deliver positive clinical outcomes. Moreover, this treatment can be a minimally invasive therapeutic option for patients who are ineligible for surgery. However, their promising outcomes for a shorter duration (3 months-24 months) must sustain for the long term of more than two years compared to the available conventional treatments. Hence, the use of BMAC or AD-MSCs therapies must be thoroughly discussed between the physician and the patient before proposing them as a firstline therapeutic approach to avoid surgery.

However, increasing the number of treatment options for knee OA does not always intend to improve the standard of care, especially when there is a lack of enough comparative trials that determine the effectiveness of a novel treatment compared to established ones.

\section{Limitations}

It is possible that BMAC and AD-MSCs injections could deliver positive outcomes in treating knee osteoarthritis, according to the results from our study. Nonetheless, the factors affecting the outcomes are but not limited to the lack of control group, a small number of studies and cointerventions, a small sample size, lack of long-term follow-up of not more than two years, the possibility of bias, and lack of objective assessment on the interventions

Although these above findings provide encouraging results, the lack of comparative study with corticosteroids and hyaluronic acid limits definitive conclusions, furthermore, the relationship of sex, age, and the severity of knee osteoarthritis could not be figured out clearly.

Additionally, MRI evaluation was not performed in all the studies to complement the clinical parameters, including the quantification of knee cartilage regeneration following the treatment. Moreover, there is a lack of comparison among the outcomes for different KL grades. Hence, more studies are required to confirm the positive long-term effects of $\mathrm{AD}$ MSCs and BMAC therapies for knee osteoarthritis.

Despite having all these limitations, the treatment of knee osteoarthritis with BMAC and AD-MSCs seems to be safe by delivering positive clinical outcomes. This treatment can be a potential minimally invasive option for those who are ineligible for invasive approaches.

\section{Conclusion}

BMAC and AD-MSCs injections prove safer and more efficacious in treating knee osteoarthritis on a short-term duration (3 months-24 months) without any adverse side effects. However, only very few randomized control studies are published to support this result. Additionally, there is a lack of high-quality research studies for more than 2 years with varied trial settings.

\section{List of abbreviations}

\begin{tabular}{|l|l|}
\hline Abbreviations & Full form \\
\hline BMAC & Bone marrow aspirate concentrate \\
\hline SVF & Stromal vascular fraction \\
\hline AD-MSCs & Adipose-Derived Mesenchymal Stem Cells \\
\hline PRP & Platelet-rich Plasma \\
\hline EMBASE & Excerpta Medica dataBASE \\
\hline RCT & Randomized Controlled Trial \\
\hline NSAIDs & Non-Steroidal Anti-Inflammatory Drugs \\
\hline HA & Hyaluronic Acid \\
\hline WOMAC & Western Ontario and McMaster Universities Osteoarthritis Index \\
\hline
\end{tabular}




\begin{tabular}{|l|l|}
\hline KOOS & Knee Injury and Osteoarthritis Outcome Score \\
\hline PRISMA & International Knee Documentation Committee \\
\hline MRI & Preferred Reporting Items for Systematic Reviews and Meta-Analyses \\
\hline ROM & Magnetic Resonance Imaging \\
\hline VAS & Range of Motion \\
\hline KSS & Visual Analogue Scale \\
\hline ICOAP & Knee Society Score \\
\hline NPS & Intermittent and Constant Osteoarthritis Pain Score \\
\hline LEFS & Neuropathic Pain Scale \\
\hline ICRS & Low Extremity Functional Score \\
\hline OKS & International Cartilage Repair Society \\
\hline NRS & Oxford Knee Score \\
\hline QoL & Numerical Rating Scale \\
\hline HTO & Quality of Life \\
\hline AM & High Tibial Osteotomy \\
\hline MFAT & Arthroscopic Microfracture \\
\hline TKA & Microfragmented adipose tissue \\
\hline
\end{tabular}

\section{Declarations}

Ethics approval and consent to participate: Not Applicable

Consent for publication: Not Applicable

Availability of data and materials: Not Applicable

Competing Interests: The authors declare that they have no competing interests

Acknowledgements: We would like to extend our heartfelt gratitude to James Corcoran, Direct Medica Stem Cells and Medica Pain Management clinic for his support and encouragement.

\section{Funding: None}

Data availability: PubMed, Scopus, Google Scholar, EMBASE, and Cochrane Library published from March 31, 2021. The authors reviewed and tabulated data according to the year of study and journal, study type, study population, therapy protocol, outcome measures, and interpretation.

Author Contribution: PP recommended the title and outline of the paper. MJS evaluated the papers included in the present review and drafted the systematic review article. PP and ST carried out the final editing of the paper. All authors read and approved the final systematic review article.

\section{Conception and design: PP}

Administrative support: $\mathrm{PP}$

Provision of study materials or patients: $\mathrm{PP}$

Collection and assembly of data: MJS

Data analysis and interpretation: MJS

Manuscript writing: MJS

Final approval of manuscript: All authors.

\section{References}

1. Kloppenburg M, Berenbaum F. (2020) Osteoarthritis year in review 2019: Epidemiology and therapy. Osteoarthr Cartil. 28: 242-248.

2. Bortoluzzi A, Furini F, Scirè CA. (2018) Osteoarthritis and its management- Epidemiology, nutritional aspects and environmental factors. Autoimmun Rev. 1097-1104.

3. Moghimi N, Rahmani K, Delpisheh A, Saidi A, Azadi NA, et al. (2019) Risk factors of knee osteoarthritis: A case-control study. Pakistan J Med Sci. 35: 636-640.

4. Loeser RF, Goldring SR, Scanzello CR, Goldring MB. (2012) Osteoarthritis: a disease of the joint as an organ. Arthritis Rheum. 64:1697-707.

5. Park H, Kim H, Lee Y. (2020) Knee osteoarthritis and its association with mental health and health-related quality of life: A nationwide cross-sectional study. Geriatr Gerontol Int. 20: 379-383.

6. Anandacoomarasamy A, March L. (2010) Current evidence for osteoarthritis treatments. Ther Adv Musculoskelet Dis; 2:1728.

7. Fodor PB, Paulseth SG. (2016) Adipose-Derived Stromal Cell (ADSC) Injections for Pain Management of Osteoarthritis in the Human Knee Joint. Aesthet Surg J. 36(2):229-236.

8. Wehling P, Evans C, Wehling J, et al. (2017) effectiveness of intra-articular therapies in osteoarthritis: a literature review. Ther Adv Musculoskelet Dis. 9(8): 183-196.

9. Laudy AB, Bakker EW, Rekers M, Moen MH. (2015) Efficacy of platelet-rich plasma injections in osteoarthritis of the knee: a systematic review and meta-analysis. $\mathrm{Br} \mathrm{J}$ Sports Med. 49(10):657-672.

10. Rabago D, Patterson JJ, Mundt M, Zgierska A, Fortney L, et al. (2014) Dextrose and morrhuate sodium injections (prolotherapy) for knee osteoarthritis: a prospective open-label trial. J Altern Complement Med. 20: 383-391. 
11. A. M. DiMarino, A. I. Caplan, and T. L. (2013) Bonfield. Mesenchymal stem cells in tissue repair. Frontiers in Immunology. 4:201.

12. Caplan AI, Correa D. (2011) The MSC: an injury drugstore. Cell Stem Cell. 9(1):11-15.

13. Caplan AI. (2007) Adult mesenchymal stem cells for tissue engineering versus regenerative medicine. Journal of Cellular Physiology. 213(2):341-347.

14. Filardo G, Madry H, Jelic M, Roffi A, Cucchiarini M, and Kon E. (2013) Mesenchymal stem cells for the treatment of cartilage lesions: from preclinical findings to clinical application in orthopaedics. Knee Surgery, Sports Traumatology, Arthroscopy. 21(8):1717-1729.

15. Kristin S. Oliver, MD, Matthew Bayes, MD, David Crane, Chakrapani Pathikonda. (2015) Clinical outcome of bone marrow concentrates in knee osteoarthritis. Journal of Prolotherapy. 7:937-946.

16. Zhang et al. (2021) Intra-Articular Injection of Autologous Adipose-Derived Stromal Vascular Fractions for the Cartilage Repair of Grade 2 and 3 Knee Osteoarthritis: A Confirmatory Clinical Trial. Research Square. 1-24.

17. Liberati A, Altman DG, Tetzlaff J, et al. (2009) The PRISMA statement for reporting systematic reviews and meta analyses of studies that evaluate healthcare interventions: explanation and elaboration. BMJ.

18. Moher D, Liberati A, Tetzlaff J, et al. (2009) Preferred reporting items for systematic reviews and meta-analyses: the PRISMA statement. BMJ.

19. Shapiro SA, Kazmerchak SE, Heckman MG, Zubair AC, O Connor MI, et al. (2017) A Prospective, Single-Blind, PlaceboControlled Trial of Bone Marrow Aspirate Concentrate for Knee Osteoarthritis. Am J Sports Med. 45(1): 82-90.

20. Shapiro SA, Arthurs JR, Heckman MG, et al. (2019) Quantitative T2 MRI mapping and 12-month follow-up in a randomized, blinded, placebo-controlled trial of bone marrow aspiration and concentration for osteoarthritis of the knees. Cartilage. 10(4):432-443.

21. Kim J Do, Lee GW, Jung GH, Kim CK, Kim T, et al. (2014) Clinical outcome of autologous bone marrow aspirates concentrate (BMAC) injection in degenerative arthritis of the knee. Eur J Orthop Surg Traumatol. 24(8): 1505-1511.

22. Sampson S, Smith J, Vincent H, Aufiero D, Zall M, et al. (2016) Intraarticular bone marrow concentrate injection protocol: short-term efficacy in osteoarthritis. Regen Med. 11(6): 511520 .

23. Krych AJ, Nawabi DH, Farshad Amacker NA, Jones KJ, Maak TG, et al. (2016) Bone Marrow Concentrate Improves of a Scaffold Plug in the knee. Am J Sports Med. 44(1): 89-91.

24. Anz A, Hubbard R. (2020) Bone Marrow Aspirate Concentrate Is Equivalent to Platelet-Rich Plasma for the Treatment of Knee Osteoarthritis at 1 Year: A Prospective, Randomized Trial. Orthrop J Sports Med.8(2).

25. Centeno C, Pitts J, Al-Sayegh H, Freeman M. (2014) Efficacy of autologous bone marrow concentrate for knee osteoarthritis with and without adipose graft. Biomed Res Int. 2014:370621.

26. Centeno CJ, Al-Sayegh H, Bashir J, Goodyear S, Freeman MD. (2015) A dose response analysis of a specific bone marrow concentrate treatment protocol for knee osteoarthritis. BMC Musculoskelet Disord. 16:258.

27. Rodriguez-Fontan F, Piuzzi N, Kraeutler M, Pascual-Garrido C. (2018) Early Clinical Outcome of Intra-Articular Injections of Bone Marrow Aspirate Concentrate for the Treatment of Early Osteoarthritis of the Hip and Knee: A Cohort Study. PM R. 10(12):1353-1359.
28. Themistocleous G, Chloros G, Kyrantzoulis I, et al. (2018) Effectiveness of a single intraarticular bone marrow aspirate concentrate (BMAC) injection in patients with grade 3 and 4 knee osteoarthritis. Heliyon. 4(10).

29. Ryu DJ, Jeon YS, Park JS, Bae GC, Kim J, et al. (2020) Comparison of Bone Marrow Aspirate Concentrate and Allogenic Human Umbilical Cord Blood Derived Mesenchymal Stem Cell Implantation on Chondral Defect of Knee: Assessment of Clinical and Magnetic Resonance Imaging Outcomes at 2-Year Follow-Up. Cell Transplant.

30. Kristin S. Oliver, Matthew Bayes, David Crane, Chakrapani Pathikonda. (2015) Clinical Outcome of Bone Marrow Concentrate in Knee Osteoarthritis. Journal of Prolotherapy.937-946.

31. Bianchi F, Maioli M, Leonardi E, Olivi E, Pasquinelli G, et al. (2013) A new nonenzymatic method and device to obtain a fat tissue derivative highly enriched in pericyte-like elements by mild mechanical forces from human lipoaspirates. Cell Transplantation. 22: 2063-2077.

32. Oliver K, Bayes M, Crane D, Pathikonda C. (2015) Clinical outcome of bone marrow concentrate in knee osteoarthritis. J Prolotherapy.

33. Shaw B., Darrow M., and A. Derian. (2018) Short-term outcomes in treatment of knee osteoarthritis with 4 bone marrow concentrate injections. Clinical Medicine Insights: Arthritis and Musculoskeletal Disorders.

34. Vad V, Barve R, Linnell E, Harrison J. (2016) Knee osteoarthritis treated with percutaneous chondral-bone interface optimization: a pilot trial. Surgical Science. 7(1): 112.

35. Hernigou P., Auregan J. C., Dubory A., Flouzat-Lachaniette C. H., Chevallier N., and Rouard H. (2018) Subchondral stem cell therapy versus contralateral total knee arthroplasty for osteoarthritis following secondary osteonecrosis of the knee. International Orthopaedics. 42(11):2563-2571.

36. Gibbs N, Diamond R, Sekyere EO, Thomas DW. (2015) Management of knee osteoarthritis by combined stromal vascular fraction cell therapy, platelet-rich plasma and musculoskeletal exercises: a case series. J Pain Res.8: 799-806.

37. Bansal H, Comella K, Leon J, et al. (2017) Intra-articular injection in the knee of adipose derived stromal cells (stromal vascular fraction) and platelet rich plasma for osteoarthritis. J Transl Med. 15(1):141.

38. Garza J, Maria D, Palomera T, Dumanian G, Anjos S. (2015) Use of Autologous AdiposeDerived Stromal Vascular Fraction to Treat Osteoarthritis of the Knee: A Feasibility and Safety Study. J Regen Med.

39. Hong Z, Chen J, Zhang S, et al. (2019) Intra-articular Injection of Autologous Adipose-Derived Stromal Vascular Fractions for Knee Osteoarthritis: A Double-Blind Randomized Self Controlled Trial Int Orthop. 43(5):1123-1134.

40. Mautner K, Bowers R, Easley K, Fausel Z, Robinson R. (2019) Functional Outcomes Following Microfragmented Adipose Tissue Versus Bone Marrow Aspirate Concentrate Injections for Symptomatic Knee Osteoarthritis. Stem Cells Transl Med. 8(11):1149-1156.

41. Pak J. (2011) Regeneration of human bones in hip osteonecrosis and human cartilage in knee osteoarthritis with autologous adipose-tissue-derived stem cells: a case series. J Med Case Rep. 5:296

42. Pak J, Chang JJ, Lee JH, Lee SH. (2013) Safety reporting on implantation of autologous adipose tissue-derived stem cells with platelet-rich plasma into human articular joints. BMC Musculoskelet Disord. 14:337. 
43. Pak J, Lee JH, Park KS, Jeong BC, Lee SH. (2016) Regeneration of cartilage in human knee osteoarthritis with autologous adipose tissue-derived stem cells and autologous extracellular matrix. Biores Open Access. 5:192-200

44. Pintat J, Silvestre A, Magalon G, et al. (2017) Intra-articular Injection of Mesenchymal Stem Cells and Platelet-Rich Plasma to Treat Patellofemoral Osteoarthritis: Preliminary Results of a Long-Term Pilot Study. J Vasc Interv Radiol. 28(12):17081713.

45. Yokota N, Yamakawa M, Shirata T, Kimura T, Kaneshima H. (2017) Clinical results following intra-articular injection of adipose-derived stromal vascular fraction cells in patients with osteoarthritis of the knee. Regen Ther. 6:108-112.

46. Hudetz D, Borić I, Rod E, Jeleč Ž, Radić A, et al. (2017) The Effect of Intra-articular Injection of Autologous Microfragmented Fat Tissue on Proteoglycan Synthesis in Patients with Knee Osteoarthritis. Genes (Basel). 8: 270

47. Pers Y-M, Rackwitz L, Ferreira R, Pullig O, Delfour C, et al. (2016) Adipose Mesenchymal Stromal Cell-Based Therapy for Severe Osteoarthritis of the Knee: A Phase I Dose-Escalation Trial. Stem Cells Transl Med. 5: 847-856.

48. Berman et al. (2019) Prospective Study of Autologous Adipose Derived Stromal Vascular Fraction Containing Stem Cells for the Treatment of Knee Osteoarthritis. Int J Stem Cell Res Ther. 6(1):064.

49. Lapuente et al. (2020) Intra-articular infiltration of adiposederived stromal vascular fraction cells slows the clinical progression of moderate-severe knee osteoarthritis: hypothesis on the regulatory role of intra-articular adipose tissue. Journal of Orthopaedic Surgery and Research. 15:137.

50. Simunec D, Salari H, Meyer J. (2020) Treatment of Grade 3 and 4 Osteoarthritis with Intraoperatively Separated Adipose Tissue-Derived Stromal Vascular Fraction: A Comparative Case Series. Cells. 9(9):2096.

51. Koh YG, Jo SB, Kwon OR, et al. (2013) Mesenchymal stem cell injections improve symptoms of knee osteoarthritis. Arthrosc J Arthrosc Relat Surg Of Publ Arthroscopy Assoc N Am Int Arthrosc Assoc. 29:748-755.

52. Koh YG, Kwon OR, Kim YS, Choi YJ. (2014) Comparative outcomes of open-wedge high tibial osteotomy with plateletrich plasma alone or in combination with mesenchymal stem cell treatment: a prospective study. Arthroscopy; 30:14531460.

53. Koh YG, Choi YJ, Kwon OR, Kim YS. (2014) Second-look arthroscopic evaluation of cartilage lesions after mesenchymal stem cell implantation in osteoarthritic knees. Am J Sports Med. 42:1628-1637.

54. Koh YG, Choi YJ, Kwon SK, Kim YS, Yeo JE. (2015) Clinical results and second-look arthroscopic findings after treatment with adipose-derived stem cells for knee osteoarthritis. Knee Surg Sports Traumatol Arthrosc. 23:1308-1316.

55. Roato I., Belisario DC, Compagno M., et al. Concentrated adipose tissue infusion for the treatment of knee osteoarthritis: clinical and histological observations. International Orthopaedics. 43(1):15-23.
56. Jones IA, Wilson M, Togashi R, Han B, Mircheff AK, and C. Thomas Vangsness JR. (2018) A randomized, controlled study to evaluate the efficacy of intra-articular, autologous adipose tissue injections for the treatment of mild-to-moderate knee osteoarthritis compared to hyaluronic acid: a study protocol. BMC Musculoskeletal Disorders. 19(1):383.

57. Bui KH-T, Duong TD, Nguyen NT, et al. (2014) Symptomatic knee osteoarthritis treatment using autologous adipose derived stem cells and platelet-rich plasma: a clinical study. Biomedical Research and Therapy. 1(1):2.

58. Nguyen PD, TD-X. Tran, Nguyen, H. T.-N., et al. (2017) Comparative clinical observation of arthroscopic microfracture in the presence and absence of a stromal vascular fraction injection for osteoarthritis. Stem Cells Translational Medicine. 6(1):187-195.

59. Kim YS, Choi YJ, Koh YG. (2015) Mesenchymal stem cell implantation in knee osteoarthritis: an assessment of the factors influencing clinical outcomes. Am J Sports Med. 43:22932301.

60. Kim YS, Choi YJ, Suh DS et al. (2015) Mesenchymal stem cell implantation in osteoarthritic knees: is fibrin glue effective as a scaffold? Am J Sports Med. 43:176-185.

61. Di Matteo B. and Kon E. (2019) Editorial commentary: biologic products for cartilage regeneration-time to redefine the rules of the game? Arthroscopy: The Journal of Arthroscopic \& Related Surgery. 35(1):260-261.

62. Hadley CJ, Shi WJ, Murphy H., Tjoumakaris FP, Salvo JP, and Freedman KB. (2019) The clinical evidence behind biologic therapies promoted at Annual Orthopaedic meetings: a systematic review. Arthroscopy: The Journal of Arthroscopic \& Related Surgery. 35(1):251-259.

63. Hong Z, Chen J, Zhang S, et al. (2019) Intra-articular injection of autologous adipose-derived stromal vascular fractions for knee osteoarthritis: a double-blind randomized self-controlled trial. International Orthopaedics. 43(5):1123-1134.

64. Gimble JM, Katz AJ, and Bunnell BA. (2007) Adipose-derived stem cells for regenerative medicine. Circulation Research. 100(9);1249-1260.

65. Zuk PA, Zhu M., Ashjian P et al. (2002) Human adipose tissue is a source of multipotent stem cells. Molecular Biology of the Cell. 13(12):4279-4295.

66. Aust L, Devlin B, Foster SJ et al. (2004) Yield of human adipose derived adult stem cells from liposuction aspirates. Cytotherapy. 6(1):7-14.

67. Oedayrajsingh-Varma MJ, Van Ham SM, Knippenberg M. et al. (2006) Adipose tissue-derived mesenchymal stem cell yield and growth characteristics are affected by the tissue-harvesting procedure. Cytotherapy. 8(2):166-177.

68. Pak J, Lee JH, Kartolo WA, Lee SH. (2016) Cartilage Regeneration in Human with Adipose Tissue-Derived Stem Cells: Current Status in Clinical Implications. Biomed Res Int. 4702674.

69. Taniguchi Y, Yoshioka T, Sugaya H, Gosho M, Aoto K, et al. (2019) Growth factor levels in leukocyte-poor platelet-rich plasma and correlations with donor age, gender, and platelets in the Japanese population. J Exp Orthop. 